\title{
Palliative treatment of intestinal obstruction in patients with gynecologic malignancies - single center experience
}

\author{
Joanna Kacperczyk-Bartnik, Aleksandra Helena Symonides, Pawel Bartnik, \\ Agnieszka Dobrowolska-Redo, Ewa Romejko-Wolniewicz, Krzysztof Czajkowski, Pawel Derlatka \\ $2^{\text {nd }}$ Chair and Department of Obstetrics and Gynecology, Medical University of Warsaw, Poland
}

\begin{abstract}
Objectives: One of the common symptoms in patients with advanced gynecologic tumors is intestinal obstruction. Palliative management may include pharmacological treatment, stenting as well as surgical removal of obstruction cause. Selection of appropriate treatment should be based on careful and individual assessment of advantages, disadvantages and possible complications. The aim of the study was to analyze the effectiveness of non-invasive treatment in patients with gynecologic malignancies suffering from intestinal obstruction.

Material and methods: It was a retrospective analysis of factors associated with primary non-invasive intestinal obstruction treatment effectiveness. Data were collected from medical records of 17 patients managed and followed-up in a single gynecologic oncology center due to endometrial cancer, fallopian tube cancer, uterine leiomyosarcoma, and ovarian cancer admitted to the ward because of symptomatic intestinal obstruction. Mean observation time lasted 40.6 months. Non-invasive treatment included fluid therapy, dexamethasone, buscolysin, mebeverine, ranitidine, simethicone, omeprazole, magnesium sulphate, semi-liquid diet, and parenteral nutrition. Characteristics including age, BMI, comorbidities, oncological treatment, histology type, stage, grade, presence of ascites, location of primary tumor and metastases were analyzed.

Results: The number of obstruction episodes varied from 1 to 5 . Mean time between multiple episodes lasted 3.2 months. 5 patients required surgical treatment. For the rest of the patients primary non-invasive treatment was sufficient.

Conclusions: Most cases of bowel obstruction in patients with advanced gynecologic malignancies can be successfully managed without invasive treatment. Moreover, non-invasive obstruction management can be applied multiple times in case of recurrence.

Key words: conservative treatment; intestinal obstruction; gynecologic neoplasms; ovarian neoplasms; palliative therapy; surgical procedures, operative
\end{abstract}

Ginekologia Polska 2019; 90, 9: 496-499

\section{INTRODUCTION}

Palliative care is an integral aspect of oncological treatment. Increasing the quality of life, soothing pain and reducing symptoms of advanced disease is crucial from both medical and ethical perspective [1, 2]. Selection of appropriate treatment should be based on careful and individual assessment of both advantages and disadvantages of available methods, together with possible complications [3-5]. Intestinal obstruction is one of the most common symptoms affecting even every second patient with advanced gynecologic tumors located in the pelvis [6]. Reported incidence of this complication among ovarian cancer patients varies between 20 and 50\% [7, 8].
The etiology of intestinal obstruction in this group of patients can be multifactorial - resulting from mass excess and intestinal infiltration, or oncological therapy side effects. Palliative management may include pharmacological treatment, stenting as well as surgical removal of obstruction cause. Apart from relieving the symptoms, obstruction treatment is vital for patient's proper nutritional status as malnutrition affects over half of ovarian cancer patients and may develop into cancer cachexia syndrome, a direct cause of death during oncological treatment $[9,10]$. Various studies claim that in case of appropriately selected therapy, pharmacological and surgical treatment are similarly effective $[11,12]$. 


\section{Objectives}

The aim of the study was to analyze which patients with advanced gynecological malignancies may benefit from primary non-invasive treatment, and which require further surgical approach.

\section{MATERIAL AND METHODS}

It was a retrospective analysis of factors associated with non-invasive intestinal obstruction treatment effectiveness. Data were collected from medical records of patients managed and followed-up in a single 14-bed gynecologic oncology center. Inclusion criteria were as follows: admission to the ward caused by symptomatic intestinal obstruction and application of primary pharmacological treatment between 2014-2016 resulting in discharge in stable general condition. In case of no improvement after 7 days of non-invasive treatment or intensification of symptoms, initially conservative treatment was considered ineffective and surgical approach was introduced. Exclusion criteria were: intestinal obstruction as a primary complaint leading to initial neoplasm diagnosis followed by the radical surgery.

The non-invasive obstruction treatment protocol included fluid therapy, dexamethasone, buscolysin, mebeverine, ranitidine, simethicone, omeprazole, magnesium sulphate and semi-liquid diet. Patients not tolerating enteral nutrition were qualified for parenteral nutrition according to the European Society for Clinical Nutrition and Metabolism (ESPEN) guidelines [13]. Patients' characteristics including past medical history, comorbidities, age at malignancy diagnosis, time from disease onset, BMI during diagnosis and each obstruction episode, body mass loss, type of applied oncological treatment, histology result, stage, grade, location of primary tumor and metastases, ascites presence, number of obstruction episodes, and type of obstruction management were analyzed. Mean observation time lasted 40.6 months (3-168 months). The FIGO (International Federation of Gynecology and Obstetrics) system was used for ovarian cancer, fallopian tube cancer, endometrial cancer and uterine sarcoma staging [14-16]. Out of 20 patients initially enrolled in the study, 17 met the criteria required for further analysis, presenting altogether 30 episodes of intestinal obstruction. All patients excluded from the study were diagnosed with serous ovarian cancer stage IIIC, grade 3 and underwent radical surgery shortly after the obstruction episode.

\section{RESULTS}

Among analyzed 17 patients 1 suffered from endometrial cancer, 2 from fallopian tube cancer, 1 from uterine leiomyosarcoma, and 13 had ovarian cancer: 10 patients serous ovarian cancer, 2 patients endometrioid ovarian cancer, 1 patient clear-cell ovarian cancer (Fig. 1). All patients suffered from

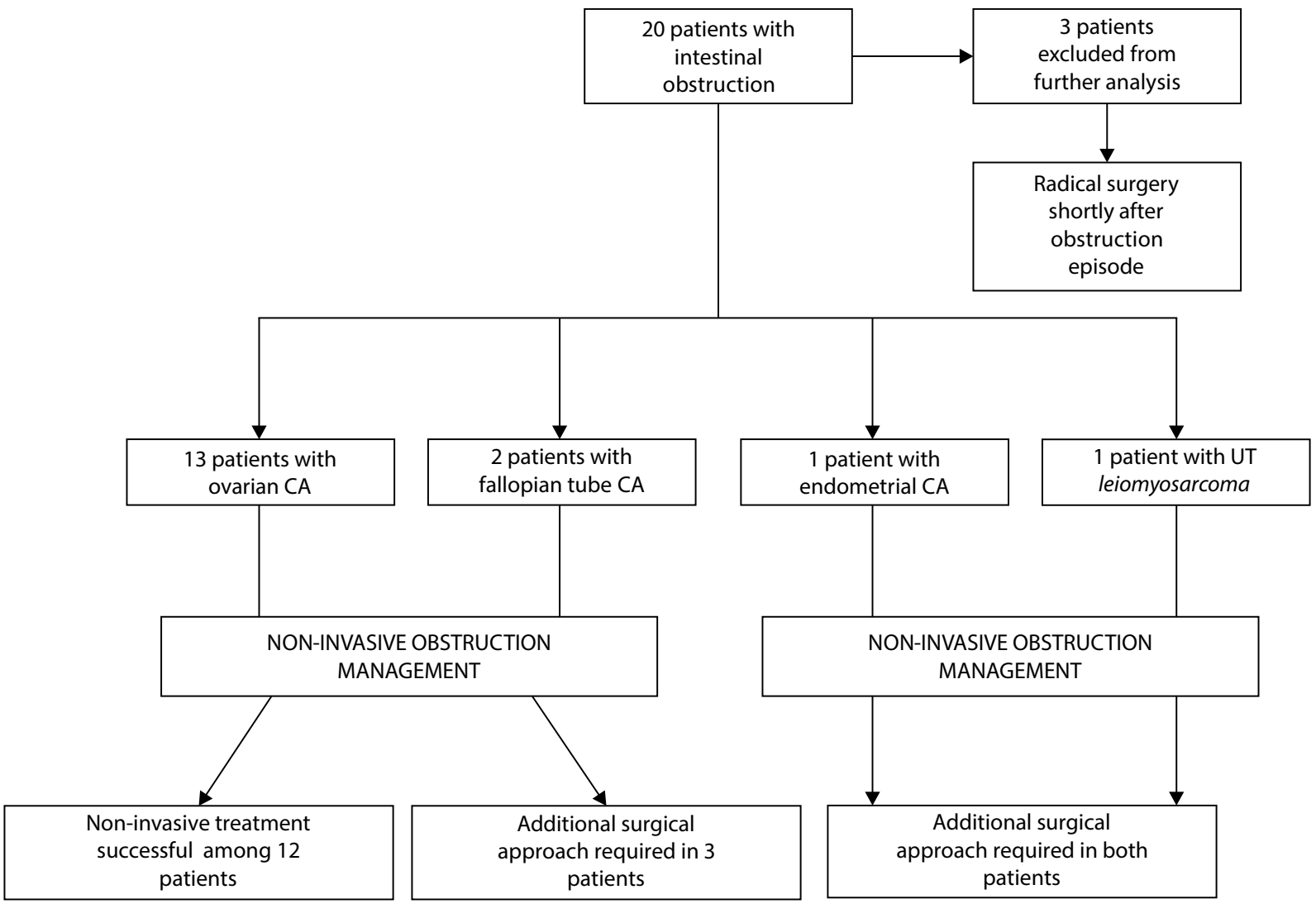

Figure 1. Intestinal obstruction in patients with gynecological malignancies — management and outcome 
poorly differentiated G3 tumors at stage III-IV. The number of applied chemotherapy lines varied between 1 and 9. Because of advanced disease 3 patients were managed without radical oncological surgery. The number of obstruction episodes varied between 1 and 5 . Mean time between multiple episodes lasted 3.2 months ( 2 weeks -22 months). In 5 (29.4\%) patients non-invasive treatment was ineffective and additional surgical approach was required. Operational interventions included: 1 repeated debulking surgery, 1 colostomy, and 3 adhesion removal surgeries. The rest of patients were successfully managed non-invasively. Patient treated with colostomy developed 4 obstruction episodes following the procedure, all of them successfully treated pharmacologically. Table 1. shows a comparison of patients' characteristics depending on the needed therapy mode. Previous abdominal surgeries included Cesarean sections, appendectomies and cholecystectomies.

\section{DISCUSSION}

Symptoms of over $70 \%$ of patients with stage III gynecologic malignancy analyzed in this study were successfully relieved with supportive treatment. Because of the size of the studied population it is difficult to obtain statistically significant evidence helpful in prediction of patients at risk of irresponsiveness to non-invasive management. For the same reason the power of statistical tests was decreased. Nevertheless, certain clinical observations were made.

First of all, patients with no history of radical surgical treatment were more likely to develop obstruction requiring invasive management. Similar conclusions were made by Bryan et al. [17] as in their study more surgical interventions were needed by patients who had been suboptimally debulked rather than optimally. Another aspect of past surgical history concerns abdominal interventions performed before oncological diagnosis. Patients who required operational management more frequently had undergone a C-section, cholecystectomy or appendectomy in the past. In all of these cases intestinal obstruction was associated with massive abdominal adhesions.

Another observation was made concerning the presence of ascites. In the studied group patients with recurrent ascites were more responsive to non-invasive obstruction management. This could be caused by an increased subjective feeling of obstruction and presentation of accompanying symptoms in response to relatively less advanced condition due to the presence of additional fluid excess. Whatever the reason, this finding gives hope to patients with ascites as it is known to be a risk factor for poor prognostic surgery outcomes [18]. Therefore, initially supportive management in this group of patients should be preferred.

Additional consideration of obstruction management outcome concerns the time of observation. Patients treated
Table 1. Patients' characteristics depending on the needed therapy mode

\begin{tabular}{|l|l|l|}
\hline Feature & $\begin{array}{l}\text { Non-invasive } \\
\text { treatment (n= 12) }\end{array}$ & $\begin{array}{l}\text { Invasive } \\
\text { treatment (n=5) }\end{array}$ \\
\hline Ovarian cancer (\%) & $11(92 \%)$ & $2(40 \%)$ \\
\hline Fallopian tube cancer (\%) & $1(8 \%)$ & $1(20 \%)$ \\
\hline Endometrial cancer (\%) & 0 & $1(20 \%)$ \\
\hline Uterine leiomyosarcoma (\%) & 0 & $1(20 \%)$ \\
\hline $\begin{array}{l}\text { Mean age at cancer } \\
\text { diagnosis (years) }\end{array}$ & $63.8(48-73)$ & $65(59-79)$ \\
\hline $\begin{array}{l}\text { Time of observation since } \\
\text { cancer diagnosis (months) }\end{array}$ & $40.7(3-168)$ & $47(15-120)$ \\
\hline $\begin{array}{l}\text { Time of 1st obstruction } \\
\text { episode since cancer } \\
\text { diagnosis (months) }\end{array}$ & $39.2(8-168)$ & $27.8(3-84)$ \\
\hline $\begin{array}{l}\text { Mean no. of obstruction } \\
\text { episodes during } \\
\text { observation }\end{array}$ & $1.5(1-3)$ & $1.8(1-5)$ \\
\hline $\begin{array}{l}\text { Abdominal surgeries } \\
\text { before oncological } \\
\text { treatment (\%) }\end{array}$ & $2(17 \%)$ & $3(60 \%)$ \\
\hline Ascites at cancer diagnosis (\%) & $9(75 \%)$ & $2(40 \%)$ \\
\hline Radical surgery (\%) & $11(92 \%)$ & $3(60 \%)$ \\
\hline $\begin{array}{l}\text { Mean no. of chemotherapy } \\
\text { lines }\end{array}$ & $3.75(1-9)$ & $2.6(2-4)$ \\
\hline $\begin{array}{l}\text { Anemia during } \\
\text { chemotherapy treated with } \\
\text { blood transfusion (\%) }\end{array}$ & $7(58 \%)$ & $3(60 \%)$ \\
\hline $\begin{array}{l}\text { Obesity at cancer diagnosis } \\
\text { Body mass loss over 5\% (\%) }\end{array}$ & $6(50 \%)$ & $2(40 \%)$ \\
\hline & $3(60 \%)$ \\
\hline
\end{tabular}

invasively presented the first episode of obstruction sooner after the cancer diagnosis than patients treated successfully in supportive manner. On the other hand, surgical patients were characterized by longer observation time following the first obstruction symptoms, which suggests their condition could be more acute, but at that time less oncologically advanced in comparison to the group responsive to pharmacological treatment. Of course, the etiology of indication for surgical treatment is essential in this context, as patients with adhesions are reported to present much longer interval in readmission for bowel obstruction than patients with malignant cause [19]. Similarly, in the study by Sartori et al. [20] patients who underwent surgical treatment showed better survival than conservatively treated group. However, opposite findings were presented in the study by Tran et al. as authors observed no statistically significant differences in outcome depending on the type of intervention - surgical, pharmacological or stent placement [21].

Since no official guidelines for management of intestinal obstruction in oncogynecological patients have been proposed till date, the first-line supportive treatment varies among cancer centers. In the study by Mangili et al. [7] 
comparing medical and surgical approach, pharmacological treatment included different doses of octreotide. The initial management described by Bais et al. [22] consisted of nasogastric tube placement, rectal enemas and intravenous fluid administration, however it was always preceding the surgical intervention. Similar management was presented in the conservative treatment implemented by Suidan et al. [8], which included bowel rest, gastrostomy or jejunostomy tube placement and intravenous fluid administration - the surgery was performed only if the conservative treatment of obstruction symptoms was unsuccessful.

\section{CONCLUSIONS}

As this study shows, most cases of bowel obstruction in advanced gynecologic malignancies can be successfully managed without invasive treatment. Individual assessment of response to therapy should be applied each time in order to achieve symptoms relief and decide which patient requires more invasive approach.

\section{REFERENCES}

1. Karlin D, Phung P, Pietras C. Palliative care in gynecologic oncology. Curr Opin Obstet Gynecol. 2018; 30(1): 31-43, doi: 10.1097/GCO.0000000000000426, indexed in Pubmed: 29227301.

2. Segev $Y$, Segev L, Schmidt M, et al. Palliative care in ovarian carcinoma patients-a personalized approach of a team work: a review. Arch Gynecol Obstet. 2017; 296(4):691-700, doi: 10.1007/s00404-017-4484-8, indexed in Pubmed: 28803353.

3. Bateni SB, Bold RJ, Meyers FJ, et al. Comparison of common risk stratification indices to predict outcomes among stage IV cancer patients with bowel obstruction undergoing surgery. J Surg Oncol. 2018; 117(3): 479-487, doi: 10.1002/jso.24866, indexed in Pubmed: 29044598.

4. Down CJ, Kumar L, Singh S, et al. A unique complication of self-expandable metal stent placement in malignant duodenal obstruction. J Surg Case Rep. 2017; 2017(9): rjx169, doi: 10.1093/jscr/rjx169, indexed in Pubmed: 28928926.

5. Takao A, Tabata T, Koizumi K, et al. Fracture of a Colonic Self-expandable Metallic Stent in Malignant Colonic Obstruction. Intern Med. 2018; 57(3): 329-332, doi: 10.2169/internalmedicine.9023-17, indexed in Pubmed: 29033426.

6. Kucukmetin A, Naik R, Galaal K, et al. Palliative surgery versus medical management for bowel obstruction in ovarian cancer. Cochrane Database Syst Rev. 2010(7): CD007792, doi: 10.1002/14651858.CD007792. pub2, indexed in Pubmed: 20614464
7. Mangili G, Aletti G, Frigerio L, et al. Palliative care for intestinal obstruction in recurrent ovarian cancer: a multivariate analysis. Int J Gynecol Cancer. 2005; 15(5): 830-835, doi: 10.1111/j.1525-1438.2005.00144.x, indexed in Pubmed: 16174232.

8. Suidan RS, He W, Sun CC, et al. Treatment Patterns, Outcomes, and Costs for Bowel Obstruction in Ovarian Cancer. Int J Gynecol Cancer. 2017; 27(7): 1350-1359, doi: 10.1097/IGC.0000000000000998, indexed in Pubmed: 28574929.

9. Balogun N, Forbes A, Widschwendter $M$, et al. Noninvasive nutritional management of ovarian cancer patients: beyond intestinal obstruction. Int J Gynecol Cancer. 2012; 22(6): 1089-1095, doi: 10.1097/IGC.0b013e318256e4d3, indexed in Pubmed: 22688964.

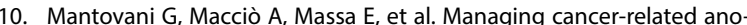
rexia/cachexia. Drugs. 2001; 61(4): 499-514, doi: 10.2165/00003495200161040-00004, indexed in Pubmed: 11324680.

11. Farias-Eisner R, Kim Y, Berek J. Surgical management of ovarian cancer. Seminars in Surgical Oncology. 1994; 10(4): 268-275, doi: $10.1002 /$ ssu.2980100407.

12. Larson J, Podczaski E, Manetta A, et al. Bowel obstruction in patients with ovarian carcinoma: Analysis of prognostic factors. Gynecologic Oncology. 1989; 35(1): 61-65, doi: 10.1016/0090-8258(89)90012-7.

13. Singer P, Berger MM, Van den Berghe $G$, et al. ESPEN Guidelines on Parenteral Nutrition: intensive care. Clin Nutr. 2009; 28(4): 387-400, doi: 10.1016/j.clnu.2009.04.024, indexed in Pubmed: 19505748.

14. Berek JS, Crum C, Friedlander M. Cancer of the ovary, fallopian tube, and peritoneum. Int J Gynaecol Obstet. 2015; 131 Suppl 2: S111-S122, doi: 10.1016/j.ijgo.2015.06.007, indexed in Pubmed: 26433667.

15. Amant F, Mirza MR, Koskas M, et al. Cancer of the corpus uteri. Int J Gynaecol Obstet. 2015; 131 Suppl 2: S96-S104, doi: 10.1016/j.ijgo.2015.06.005, indexed in Pubmed: 26433681.

16. Prat J, Mbatani,. Uterine sarcomas. International Journal of Gynecology \& Obstetrics. 2015; 131: S105-S110, doi: 10.1016/j.ijgo.2015.06.006.

17. Bryan DN, Radbod R, Berek JS. An analysis of surgical versus chemotherapeutic intervention for the management of intestinal obstruction in advanced ovarian cancer. Int J Gynecol Cancer. 2006; 16(1): 125-134, doi: 10.1111/j.1525-1438.2006.00283.x, indexed in Pubmed: 16445622.

18. Krebs HB, Goplerud DR. Surgical management of bowel obstruction in advanced ovarian carcinoma. Obstet Gynecol. 1983; 61(3): 327-330, indexed in Pubmed: 6823374.

19. Furnes $B$, Svensen $R$, Helland $H$, et al. Challenges and outcome of surgery for bowel obstruction in women with gynaecologic cancer. Int J Surg. 2016: 27: 158-164, doi: 10.1016/j.ijsu.2016.02.002, indexed in Pubmed: 26853847.

20. Sartori E, Chiudinelli F, Pasinetti B, et al. Bowel obstruction and survival in patients with advanced ovarian cancer: analysis of prognostic variables. Int J Gynecol Cancer. 2009; 19(1): 54-57, doi: 10.1111/IGC.0b013e318198ff4b, indexed in Pubmed: 19258942.

21. Tran E, Spiceland C, Sandhu NP, et al. Malignant Bowel Obstruction in Patients With Recurrent Ovarian Cancer. Am J Hosp Palliat Care. 2016; 33(3): 272-275, doi: 10.1177/1049909114566225, indexed in Pubmed: 25552305.

22. Bais J, Schilthuis MS, Slors J, et al. Intestinal obstruction in patients with advanced ovarian cancer. Int J Gynecol Cancer. 1995; 5(5): 346-350, indexed in Pubmed: 11578502. 